\title{
SeDuCe: a Testbed for Research on Thermal and Power Management in Datacenters
}

\author{
Jonathan Pastor \\ IMT Atlantique - Nantes \\ jonathan.pastor@imt-atlantique.fr
}

\author{
Jean Marc Menaud \\ IMT Atlantique - Nantes \\ jean-marc.menaud@imt-atlantique.fr
}

\begin{abstract}
With the advent of Cloud Computing, the size of datacenters is ever increasing and the management of servers and their power consumption and heat production have become challenges. The management of the heat produced by servers has been experimentally less explored than the management of their power consumption. It can be partly explained by the lack of a public testbed that provides reliable access to both thermal and power metrics of server rooms. In this paper we propose SeDuCe, a testbed that targets research on power and thermal management of servers, by providing public access to precise data about the power consumption and the thermal dissipation of 48 servers integrated in Grid'5000 as the new ecotype cluster. We present the chosen software and hardware architecture for the first version of the SeDuCe testbed, highlighting its current limitation and proposing some improvements that will increase its relevance.
\end{abstract}

Keywords-Datacenters; Scientific testbed; Thermal management; Power management; Green computing.

\section{INTRODUCTION}

The advent of web sites with a global audience and the democratization of Cloud Computing have led to the construction of datacenters all over the world. Datacenters are facilities that concentrate from a few servers up to hundreds of thousands of servers hosted in rooms specially designed to provide energy and cooling for the servers. These facilities are widely used for applications such as the hosting of web services or High Perfomance Computing.

In recent years, the size of datacenters is ever increasing, which leads to new challenges such as designing fault tolerant software to manage at large scale the servers and energy management of server rooms. On the latter challenge, many research efforts have been conducted [1] [2], most of them focusing on the implementation of on demand power management systems, such as Dynamic voltage scaling (DVFS) [3] [4] and vary-on vary-off (VOVO) [5] [6]. Some work has been made to extend existing scientific testbeds with power monitoring of experiments : for example Kwapi [7] enables researchers to track the power consumption of their experiments conducted on Grid' 5000.

On the other hand, the thermal management of servers has been less explored, a large part of the existing work considering only simulations [8]. This can be explained, partly, by the difficulty of conducting experiments involving thermal monitoring of servers : to ensure that data recorded experimentally is valid, experimentions must be conducted on a testbed that contains many temperature sensors, not only positionned on cooling systems, but also at the front and the back of each server of racks.
In addition, such a testbed must enable reproducible experimentations, by providing its users with a full control on experimental conditions of their experiments and by exposing its data in a non misleading way, via a well documented API (Application Programming Interface).

Finally, as power management and temperature management of servers are related problems [9], there is a need for a testbed that enables users to access to both thermal and power data of servers.

As far as we know, there is no public testbed that enables researchers to work on both power and thermal aspects of servers functionning. The objective of the SeDuCe project is to propose such a testbed : SeDuCe testbed enables its users to use, in the context of the ecotype cluster integrated in the Grid'5000 infrastructure [10], 48 servers located in 5 airtight racks with a dedicated central cooling system (CCS) positioned inside one of the rack. In parallel of conducting the experiment by leveraging the tools provided by Grid'5000, users can get access to thermal and power data of the testbed via a web portal and a user-friendly API. Stability of experimental conditions is guaranteed by hosting the testbed in a dedicated room equipped with a secondary cooling system (SCS) that enables a precise thermo-regulation of the environment outside the cluster. As resources of the testbed are made publicly available via the Grid' 5000 infrastructure, all its users will be able to perform reproducible research on thermal and power management of servers.

\section{TESTBED DESIGN}

A. Ecotype : a Grid'5000 cluster dedicated to the study of power and thermal management of servers

We have built the ecotype cluster, which is composed of 48 servers and has been designed for research related to power and thermal management in datacenters. The ecotype cluster is integrated in the Grid' 5000 infrastructure and is made available to any of its users : they can reserve servers of the ecotype cluster and conduct experiments on them by using the usual Grid'5000 tools. Meanwhile they can access in real time to information regarding the temperature of the servers involved in their experiments, and get the power consumption of any parts of the testbed (servers, switches, cooling systems, ...).

Each server of the ecotype cluster is a DELL PowerEdge R630 that contains a pair of Intel Xeon E5-2630L v4 CPUs (10 cores, 20 threads per CPU), 128GB of RAM, and 400GB SSD. The CPUs have been designed to have a power consumption that is lower than other CPUs of the XEON 26XX serie, with a thermal design power (TDP) of 55W. Each server is connected 
via two $10 \mathrm{GBe}$ links to the Grid' 5000 production network, and via a single $1 \mathrm{GBe}$ link to the Grid'5000 management network. Additionally, each server is certified to work in environments where temperature can be up to $35^{\circ} \mathrm{C}$.

The cluster is composed of 5 Racks (Z1, Z2, Z3, Z4, $\mathrm{Z5}$ ) and, as shown on Figure 1, one rack (Z3) is in charge of cooling the cluster by hosting a dedicated central cooling system (CCS), while remaining racks are computing racks and are dedicated to hosting servers.

As depicted by Figure 1, computing racks host 12 servers, following two layouts of server positions : one layout where servers are organised in a concentrated way with no vertical space between servers (Z1 and Z2), and a second layout where servers are spaced at $1 \mathrm{U}$ intervals ( $\mathrm{Z} 4$ and $\mathrm{Z5}$ ).

We have deliberately chosen to use these two layouts : they will enable to study the impact of a layout on the temperature and the power consumption of servers.

In addition to servers, the cluster contains three network switches that are in charge of connecting servers to production and management networks of the Grid'5000 testbed. Three racks $(\mathrm{Z2}, \mathrm{Z4}, \mathrm{Z5})$ are hosting each a network switch.

The cluster has also been designed to leverage "Schneider Electric IN-ROW" racks that create an inside airtight environment for servers, and guarantee that the environment outside the cluster has a limited impact on temperatures inside the racks. The temperature inside the cluster is regulated by central cooling system (CCS), connected to a dedicated management network, which implements an SNMP service that enables to remote control the cooling and to access its operating data.

In addition, the temperature outside the cluster is regulated by a secondary cooling system (SCS) which is mounted from the ceiling of the server room : the SCS is in charge of maintaining a constant temperature in the server room.

Finally, we have installed Airflow management panels between servers, in order to separate the air of the hot aisle from the air of the cold aisle.

\section{B. Power Monitoring}

Each element composing the cluster (servers, network switches, fans, condensators, ...) is electrically monitored, and its power consumption is recorded every second in a database.

Electrical plugs of servers and network switches are connected to power distribution units (PDUs), which in turn share power consumption of servers and network switches via a dedicated service network. Each computing racks contains two PDUs, and each server of a computing rack has two electrical plugs. As depicted in Figure 2, the electrical plugs of a server are connected to two different PDUs, which enables servers to have electrical redundancy. The power consumption of each plug of a server can be fetched by issuing an SNMP request to the PDU it is connected.

In a similar way, energy consumption of the CCS can be fetched by requesting its SNMP service. This SNMP service is able to provide an overall power consumption of the CCS and also the power consumption of each internal part such as the condensator or the fans. The SCS does not implement any built-in networking access, and thus cannot share its metrics with any component over a network. To solve this problem, we

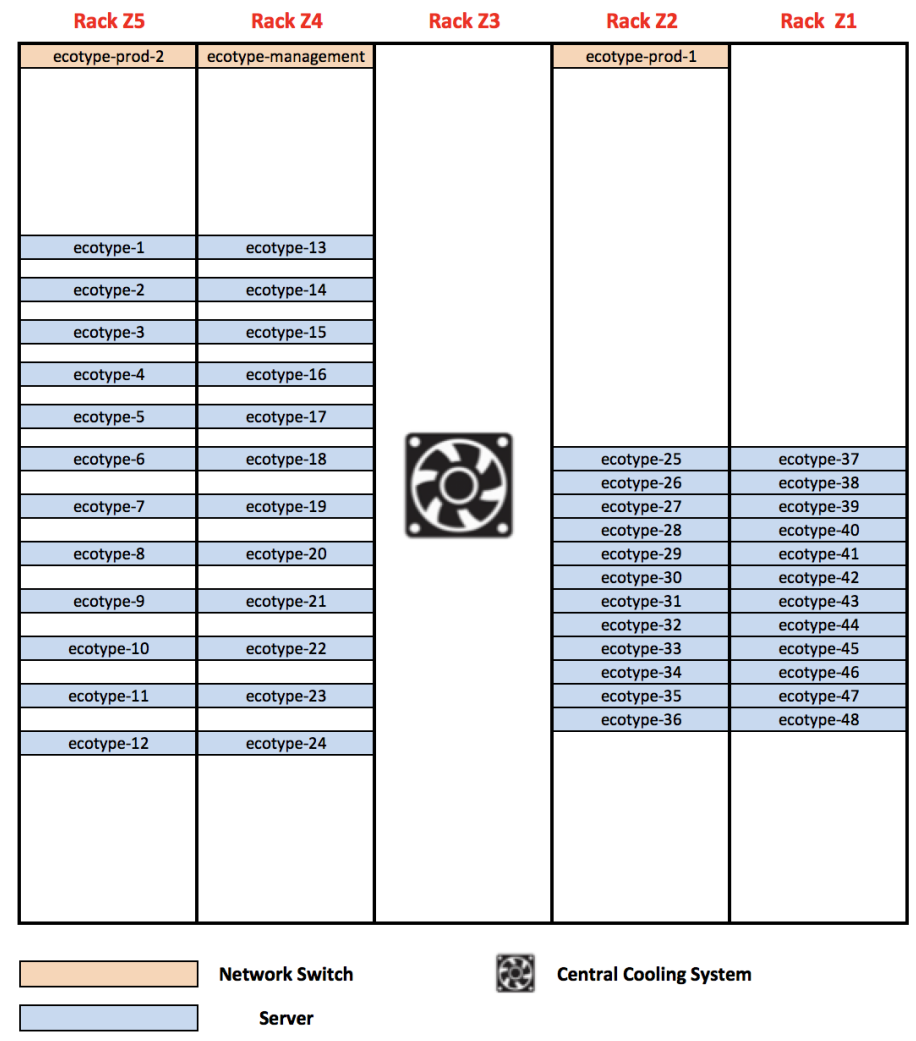

Figure 1. Layout of the ecotype cluster (front view)

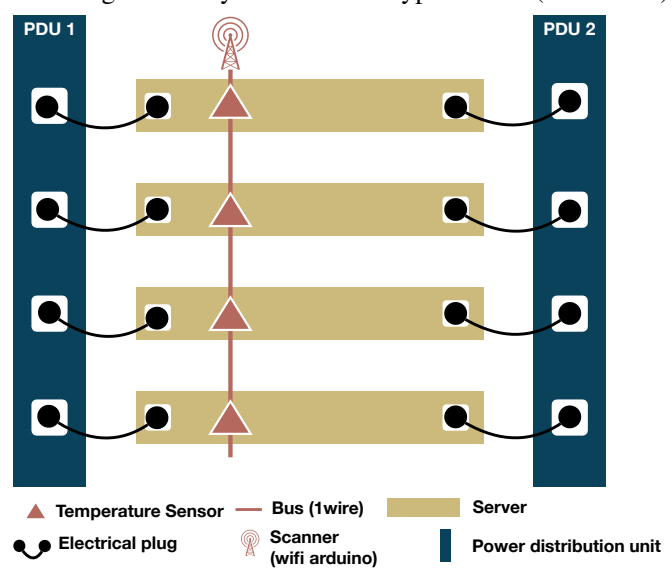

Figure 2. Back view of a server rack

instrumented several parts of the SCS by using a Fluksometer ${ }^{1}$ : a Fluksometer is a connected device that can monitor several electrical metrics (power consumption, voltage, amperage, ...) and expose their values over a network via a web-service.

Finally, we have added an additional system that tracks overall power consumption of both servers and the CCS. This additional system is based on the Socomec G50 metering board $^{2}$, an electronic board that can measure in real time the power consumption of several electrical plugs. We use the Socomec G50 for checking that the aforementioned source of power consumption data are sound. These additional metrics are fetched by using the modbus protocol.

\footnotetext{
${ }^{1}$ https://www.flukso.net/about

${ }^{2}$ https://www.socomec.com/range-communication-interfaces_en.html? product=/diris-g_en.html
} 


\section{Temperature Monitoring}

In order to track the thermal behavior of the ecotype cluster, each server is monitored by a pair of temperature sensors : one sensor is positioned at the front of the server (in the cold aisle) and another sensor is positioned at the back of the server (in the hot aisle).

As depicted by Figure 2, each temperature sensor is a member of a bus (based on the lwire protocol) connected to a Scanner (implemented by using a wifi arduino) in charge of gathering data from the temperature sensors of the bus. As the front and the back of each server is monitored by temperature sensors, computing racks have in total two Scanners and two buses : a front bus for monitoring the cold aisle and a back bus dedicated to the hot aisle. Scanners fetch temperatures from their sensors at a frequency of one reading per sensor every second.

For a first version of the SeDuCe testbed, we have chosen to use the DS18B20 sensor, a temperature sensor produced by "Maxim Integrated" 3 that costs approximately $3 \$$. According to the specifications provided by its constructor, this sensor is able to provide a temperature reading every $750 \mathrm{~ms}$ with a precision of $0.5^{\circ} \mathrm{C}$ between $-10{ }^{\circ} \mathrm{C}$ and $85^{\circ} \mathrm{C}$.

The key feature of the DS18B20 sensor is that it is able to work as part of an lwire bus. In the context of the SeDuCe infrastructure, 12 DS18B20 sensors are connected together to form an lwire bus, and a Scanner, an nodeMCU arduino with built-in wifi capabilities, fetches periodically their temperature readings. The current version of the firmware used by Scanners scans an 1wire bus every seconds, and then pushes temperature data to a Temperature Registerer service, as illustrated in Figure 3.

We also developed a contextualisation tool to generate firmwares for the Scanners. It leverages the PlatformIO framework to program a Scanner that pushes data to a web-service. Using this contextualisation tool is simple : a developer needs to define a program template in a language close to $\mathrm{C}$ language and marks some parts of code with special tags to indicate that these parts need to be contextualized with additional information, such as initializing a variable with the ID of a Scanner device or with the address of a remote web-service (such as the one that will receive temperature records). The contextualisation tool takes this program and a context as input parameters, analyses the template program, and completes parts that requires contextualisation with information provided in the context, which results in valid $\mathrm{C}$ language source file. Then, the firmware is compiled and automatically uploaded to Scanners via their serial ports. By leveraging this contextualisation tool, we can remotely configure Scanners and update their firmware.

\section{Seduce portal}

To help users to easily access power and thermal metrics generated by the SeDuCe testbed, we developed a platform that exposes publicly two components : a web portal ${ }^{4}$ and a documented API ${ }^{5}$ (Application Programming Interface).

\footnotetext{
${ }^{3} \mathrm{https} / / /$ datasheets.maximintegrated.com/en/ds/DS18B20.pdf

${ }^{4}$ https://seduce.fr

${ }^{5}$ https://api.seduce.fr/apidocs
}

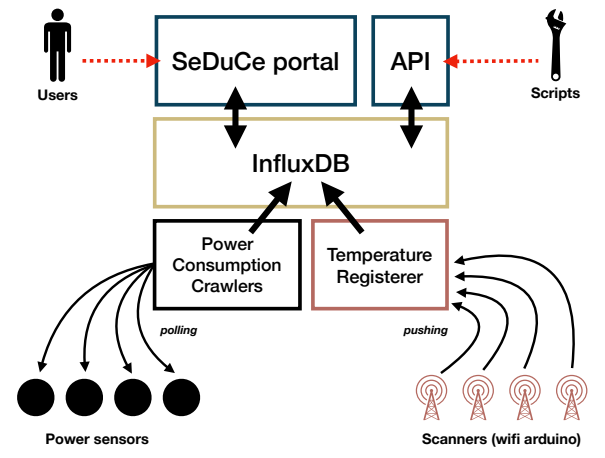

Figure 3. Architecture of the SeDuCe portal

As illustrated by Figure 3, the web portal and the API fetch data from a time series database (TSDB) based on InfluxDB ${ }^{6}$. Influx $D B$ enables to store a large quantity of immutable time series data in a scalable way. In the background, InfluxDB creates aggregates of data by grouping periodically data from a same series. These aggregated sets of data enable the web portal to promptly load data used for visualization.

Two kind of components are in charge of inserting data in the database : the Power consumption crawlers and the Temperature Registerer. Power consumption crawlers are programs that are in charge of polling data from PDUs, Socomecs, Fluksometer, the CCS and the SCS. In turn, this data is inserted in the database. On the other hand, the Temperature Registerer is a web service that receives temperature data pushed from nodeMCU arduino devices, and inserts it in the database.

The web portal and the API are both written in Python and leverage the "Flask" micro web framework. The API component makes an extensive use of the Swagger framework 7 which automatises the generation of complete REST web services and their documentations from a single description file (written in JSON or YAML). This choice has enabled us to focus on the definition and the implementation of the API, by reducing the quantity of required boilerplate code.

All the components depicted in Figure 3 are implemented as micro-services. Our system is able to register 200 metrics per seconds with minimal hardware requirements (it is currently hosted on a single computer). In the case we add more sensors to our testbed, it is likely that the existing components would be sufficient. In the case that one of the component would not be able to cope with the additional workload, it would be easy to setup an high availability approach by using a load balancer such as the Nginx web-server that can forward requests to a pool of instances of the component.

\section{Evaluating THE DS18B20 SENSOR}

In this section we evaluate the behaviour of the DS18B20 sensor in order to evaluate its fitness to the thermal measurement of the heat dissipated by servers.

\section{A. Experimental Setup}

The performance evaluation of the DS18B20 can be made regarding two criteria : the precision the temperature it reports, and its response time. The precision of a sensor corresponds to the difference between the reported temperature and the actual

\footnotetext{
${ }^{6}$ https://www.influxdata.com/

${ }^{7}$ https://swagger.io/
} 
temperature (usually it is estimated by a calibrated reference thermometer). On the other hand, the response time of a sensor corresponds to the time taken by the temperature reported by the sensor to converge to the reference temperature.

The evaluation of these two parameters requires an experimental environment and the definition of an experimental protocol.

To ensure that the validation of the DS18B20 sensor is based on reliable data, we used an Hart Scientific 7103 microbath which contained a silicon oil maintained at a specified homogeneous temperature. The temperature inside the microbath was controlled by two thermometers (a PT100 probe and a Hart Scientific 1502A). We conducted two experiments : a first experiment to evaluate the precision of the temperatures reported by DS18B20 sensors, and a second experiment to evaluate the reaction time of DS18B20 sensors.

\section{B. Sensor precision}

\begin{tabular}{|c|c|c|c|c|c|}
\hline \multirow{2}{*}{ Target } & Sensors & \multicolumn{5}{|c|}{} \\
\cline { 2 - 6 } & Sensor 1 & Sensor 3 & Sensor 3 & Sensor 4 & Sensor 5 \\
\hline $10^{\circ} \mathrm{C}$ & $10.86 \pm 0.05$ & $10.24 \pm 0.03$ & $10.06 \pm 0.06$ & $10.91 \pm 0.05$ & $10.13 \pm 0.02$ \\
$20{ }^{\circ} \mathrm{C}$ & $20.75 \pm 0.00$ & $20.00 \pm 0.02$ & $19.98 \pm 0.05$ & $20.86 \pm 0.04$ & $20.00 \pm 0.00$ \\
$30{ }^{\circ} \mathrm{C}$ & $30.74 \pm 0.04$ & $29.93 \pm 0.07$ & $29.91 \pm 0.06$ & $30.82 \pm 0.06$ & $29.97 \pm 0.06$ \\
$40{ }^{\circ} \mathrm{C}$ & $40.72 \pm 0.05$ & $39.81 \pm 0.06$ & $39.87 \pm 0.05$ & $40.77 \pm 0.06$ & $39.92 \pm 0.07$ \\
$50{ }^{\circ} \mathrm{C}$ & $50.72 \pm 0.05$ & $49.75 \pm 0.07$ & $49.85 \pm 0.06$ & $50.75 \pm 0.07$ & $49.89 \pm 0.08$ \\
$60^{\circ} \mathrm{C}$ & $60.76 \pm 0.03$ & $59.76 \pm 0.05$ & $59.88 \pm 0.03$ & $60.79 \pm 0.06$ & $59.93 \pm 0.06$ \\
$70{ }^{\circ} \mathrm{C}$ & $70.83 \pm 0.06$ & $69.79 \pm 0.06$ & $69.92 \pm 0.06$ & $70.86 \pm 0.03$ & $69.99 \pm 0.04$ \\
\hline
\end{tabular}

TABLE I. Comparison of 5 DS18B20 sensors (mean temperature \pm standard deviation)

To evaluate the precision of DS18B20 sensors, we plunged 5 sensors into a silicon oil configured to be at a precise target temperature. Once the temperature of the silicon oil is stabilized, we measured the temperatures reported by the 5 DS18B20 sensors during 20 minutes. The target temperature was initially set at $10^{\circ} \mathrm{C}$, and incrementally increased by steps of $10{ }^{\circ} \mathrm{C}$.

Table I summarizes the temperatures reported by DS18B20 sensors at each step of temperature.

First, it is noticeable that for each targeted temperature, the average temperature reported by a DS18B20 sensor is in a range of $1^{\circ} \mathrm{C}$ band around the target temperature. While this value is higher than the $0.5^{\circ} \mathrm{C}$ advertised by the manufacturer, it should be relativised : when the target temperature is set to $20^{\circ} \mathrm{C}$ the difference between the worst sensor and the reference temperature is less than $5 \%$, and at $50^{\circ} \mathrm{C}$ it becomes lower than $2 \%$. These results should also be put in the perspective of the price range of DS18B20 sensors (approximately $3 \$$ per unit).

Second, it appears that for a given sensor, the difference between the reported temperature and the reference temperature is stable for any temperature steps. Thus, by comparing several temperature records of a DS18B20 sensor with those of a reference thermometer (such as the one we used to stabilize the silicon oil), it would be possible to modelise the difference between the temperature reported by the sensor and the reference temperature. This would enable the implementation of a calibration mechanism that will be in charge of correcting the difference between the reported temperature and the reference temperature.

\section{Sensor reactivity}

To evaluate the reaction time of DS18B20 sensors, we plunged a single DS18B20 sensor from the ambient air at a temperature of $23^{\circ} \mathrm{C}$ into a silicon oil at a temperature of $30^{\circ} \mathrm{C}$. We measured the time taken by the temperature reported by the DS18B20 sensor to converge to the temperature reported by the reference thermometer. Figure 4 plots these measurements.

The sensors takes about 10 seconds to stabilize at the temperature reported by the reference thermometer. This delay can be explained by the fact the DS18B20 sensor uses a "TO92" semiconductor package which leverages plastic to protect its internal components from the external environment. This plastic shield enables the sensor to be used in a wide range of environments. However, the drawback is that it creates a thermal inertia, as the heat has to first penetrate the plastic shield before reaching the electronic part of the sensor.

This result indicates that DS18B20 are not suitable for configurations where temperature can suddenly change as the reactivity of the DS18B20 sensor is low. However, it appears to be appropriate for cases where the temperature is already stabilized and will only change smoothly, such as about a few ${ }^{\circ} \mathrm{C}$ over tens of seconds, such as in the hot aisle of the cluster.

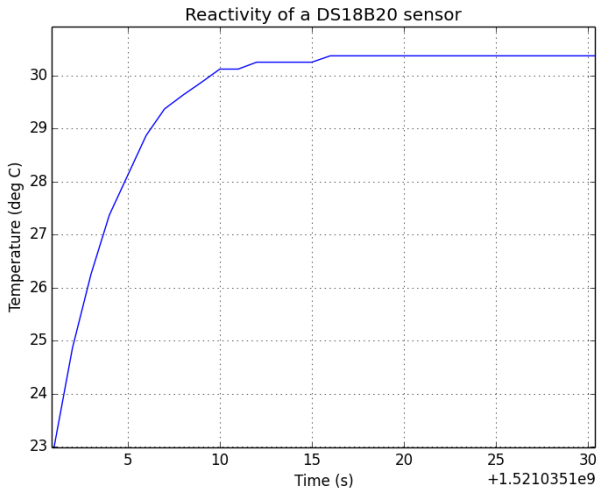

Figure 4. Reactivity of a DS18B20 plunged in a silicon oil at $30^{\circ} \mathrm{C}$ temperature

\section{EXPERIMENTATION}

To illustrate the potential of the SeDuCe platform, we conducted an experiment that mixes energetic and thermal monitoring. The goal of the experiment was to verify that the data produced by the SeDuCe testbed is reliable, by designing an experiment that will use both the thermal and the power data produced by the testbed. These data would be used to reproduce a scientifically validated observation, such as the impact of idle servers on the power consumption and the temperature of a server room.

Such experiment has been conducted in the past [9], however as far as we know there is no public testbed that would enable researchers to reproduce this result : by reproducing this result on the SeDuCe testbed, we think that it would demonstrate the soundness of our approach and the usefulness of our testbed.

\section{A. Description of the experiment}

To illustrate the scientific relevance of our testbed, we wanted to reproduce the observations made by third party publication [9]. 
In [9], authors have highlighted an interesting fact : in a datacenter idle servers (i.e. servers that are turned on while not being used to execute any workload) have a significant impact on power consumption and heat production. We decided to try to reproduce this observation.

For this experiment, servers of the ecotype cluster are divided in three sets of servers:

- Active servers: servers with an even number (ecotype2 , ecotype-4, ..., ecotype-48) were executing a benchmark that generates a CPU intensive workload.

- Idle servers: a defined quantity $(0,6,12,18,24$ servers) of servers with an odd number (ecotype-1, ecotype- $3, \ldots$, ecotype-47) was remaining idle.

- $\quad$ Turned-off servers: remaining servers were electrically turned off.

and during one hour we recorded the power consumption of the CCS and the average temperature in the hot aisle of the ecotype cluster. The CPU intensive workload was based on the "sysbench" tool : the goal was to stress CPUs of each servers, resulting in an important power consumption and a bigger dissipation of heat. To guarantee the statistical significance of the measurements, each experimental configuration was repeated 5 times, leading to a total number of 25 experiments.

We executed two sets of experiments : one with the SCS turned-on (Figure 5) and the other while the SCS was turned off (Figure 6). The objective of turning off the SCS was to identify the impact of the SCS over the CCS.

\section{B. Results}

Figure 5 plots the cumulated power consumption of the CCS and the average temperature in the hot aisle of the cluster with the SCS enabled.

First, it is noticeable that as the number of idle nodes increases, both the energy consumed by the SCS and the temperature in the hot aisle of the rack increase. This can be explained by the fact that an idle node consumes some energy and produces some heat, which increases the workload of the CCS.

The second element highlighted by Figure 5 is that the impact of idle nodes is not linear : the red line representing the CCS consumption follows an exponential pattern and the blue line representing the average temperature in the hot aisle follows a sigmoid pattern. The exponential pattern of the power consumption of the CCS can be explained by the fact that the heat produced by a given server has an impact on the temperature of surrounding servers, which causes the creation of "hot-spots". These hot-spots are difficult to cool down, and they increase the needs in terms of cooling. As the temperature of hot spots is higher that the average temperature in racks, when a sensors of the CCS detects an hot-spot, it leads the CCS to work intensively and more frequently to reduce the hot-spot. On the other hand, the sigmoid pattern of the average temperature in the hot aisle is explained by the fact that the CCS has its own monitoring of the temperature inside racks, and when temperatures at the back of servers reach a threshold, the CCS works more frequently and intensively to make the average temperature back under a specific threshold, thus attenuating the increase of the temperature in the hot aisle.
Figure 6 plots the cumulated power consumption of the CCS and the average temperature in the hot aisle of the cluster while the SCS is disabled. This figure highlights that the power consumption of the CCS is lower when the SCS is disabled. This can be explained by the fact that the SCS was configured to maintain a temperature of $19{ }^{\circ} \mathrm{C}$ in the outside room, which corresponds to the maximum temperature target of the CCS : the outside air, by means of thermal conduction, contributes to warm the air inside the racks at a temperature that activates the CCS. As a consequence, it increases the needs in term of cooling inside the cluster, leading to an higher power consumption of the CCS.

This experimental campaign has shown that idle servers have an important impact on the power consumption of cooling systems and overall racks temperature, thus it confirms the observation made in this publication [9].

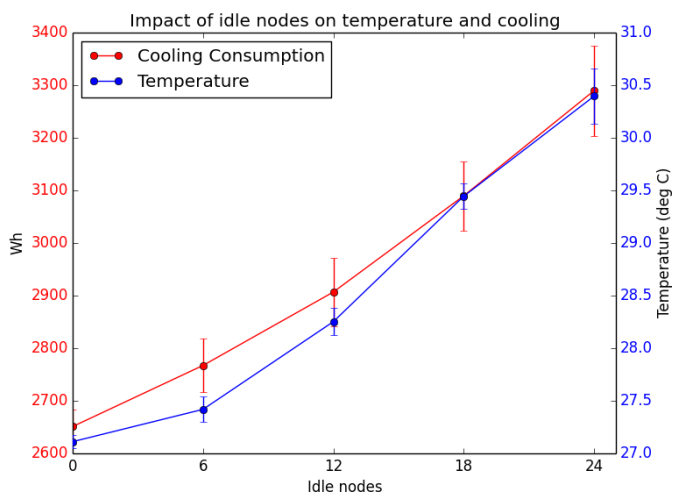

Figure 5. Central cooling consumption and average temperature in the hot aisle (SCS enabled)

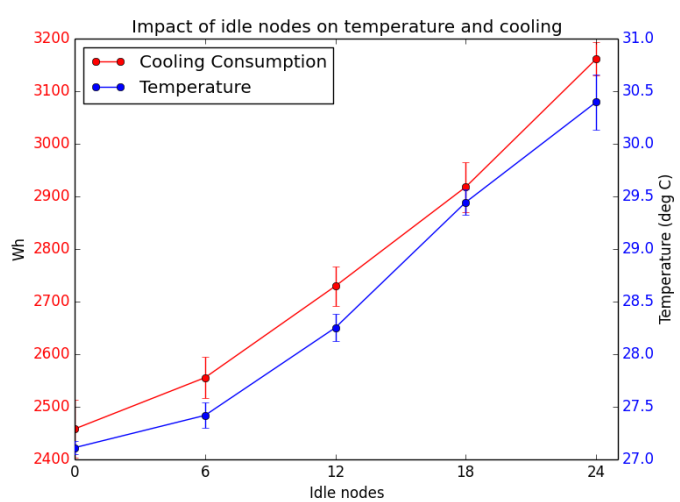

Figure 6. Central cooling consumption and average temperature in the hot aisle (SCS disabled)

\section{Increasing the reactivity of temperature sensors}

While being suitable for monitoring temperatures in the hot aisle of our cluster, we have noticed that DS18B20 sensors were experiencing difficulties track the sudden temperature changes that occur in the cold aisle when CCS is active. Figure 7 compares a DS18B20 sensor and with several thermocouple based sensors, one based on the Graphtec GL 220, and two thermocouples based on the MAX $31850 \mathrm{~K}$ card, all positioned in the cold aisle at the front of the "ecotype-42" server, and recording temperatures during a typical instance of the CCS workload. We used the GL 220 to define a reference temperature in the cold aisle. At $t=80 \mathrm{~s}$, the CCSs just finished cooling the cold aisle, thus the temperature has reached its 


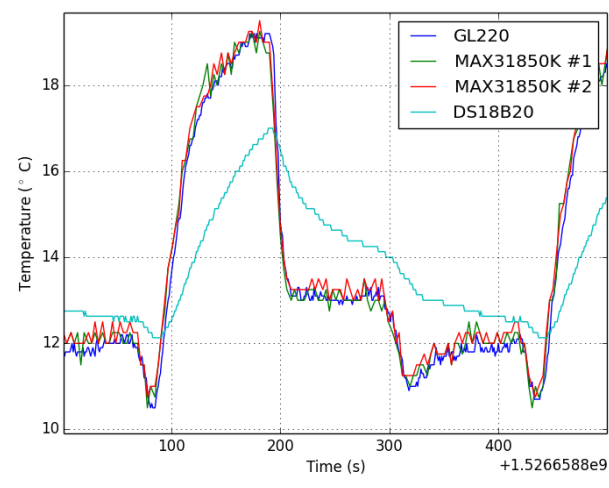

Figure 7. Comparison between DS18B20 and thermocouples

lowest level and is going to increase. The CCS restarts to cool the cluster at $t=180$ s. It is noticeable that the temperature measured by the thermocouple based sensor drops significantly after few seconds, while the DS18B20 sensor measures a temperature that is falling gradually over a hundred of seconds. Even worse, we can see at $\mathrm{t}=430 \mathrm{~s}$ that the minimal temperature measured by the DS18B20 sensor does not correspond to the minimal temperature reported by the thermocouple based sensors, but rather to the intersection between its decreasing measured temperature and the increasing temperature measured by thermocouples. We explain this observation by the thermal design of the DS18B20 : as stated in Section III-C it leverages the "TO-92" semiconductor package which leads to a significant thermal inertia in air environment. On the other hand, thermocouple based sensors have a better reactivity : they can reactively track the decrease of temperature. It is also noticeable that the temperature reported by the two MAX31850K board are very close to the reference temperature reported by the GL 220. However, the two MAX31850K we used were embedded on the ADA1727 board, manufactured by Adafruit, which has a unit cost of $14.95 \$$. In Section V we will study an approach that enables to reduce the unit cost of MAX31850K thermocouples.

\section{FUTURE WORK}

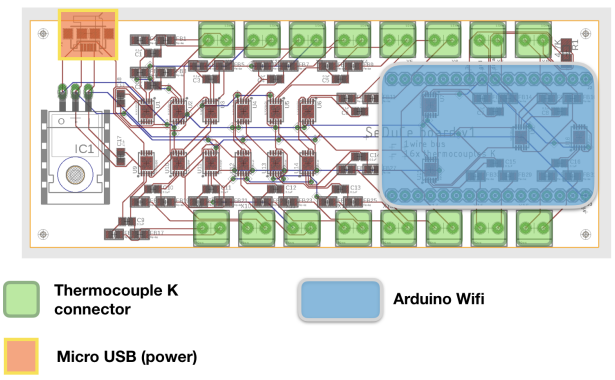

Figure 8. Prototype of the SeDuCe board

In Section IV-C, the potential of thermocouple based sensors for reactive temperature measurements has been highlighted. For the second version of the SeDuCe testbed, we have studied the replacement of DS18B20 sensors by thermocouple sensors based on the MAX31850K which is compatible with the 1wire protocol. However, the most popular electronic card featuring the MAX31850K, the ADA1727 board manufactured by Adafruit, costs $15 \$$, which 5 times more expensive than the DS18B20, and it only features a single thermocouple channel.

We have designed our own electronic board to reduce the cost of using many thermocouple based sensors. Figure 8 depicts our SeDuCe board, which features 16 thermocouples channels, based on 16 MAX31850K chips connected via an 1 wire bus. We have added 2 lines of pin female headers for pluggin a nodeMCU Arduino, and a micro USB port that supplies the board and the Arduino with electricity.

\section{CONCLUSION}

In this article we have presented our initial work on building the SeDuCe testbed, which targets research related to power and thermal management in datacenters. We have described the architecture of the testbed, which is built on buses of sensors, storage of power and thermal metrics in a time series oriented database (InfluxDB) and an user friendly web portal and a documented API. We have also detailed the components used in this first version of the SeDuCe testbed, and their limitations such as the lack of reactivity of the DS18B20 sensor, a low cost temperature sensor that shows its limits in situation where temperature changes suddendly. However, this model of sensor remains relevant in a large number of situations. Future work on this first version of the $\mathrm{SeDuCe}$ testbed will mainly focus on evaluating more reactive temperature sensors. We are thinking to replace all or a part of the existing sensors by thermocouple based sensors, whose main advantages are that they are more reactive and more precise while remaining affordable.

\section{REFERENCES}

[1] F. Hermenier, X. Lorca, J.-M. Menaud, G. Muller, and J. Lawall, "Entropy: a consolidation manager for clusters," in Proceedings of the 2009 ACM SIGPLAN/SIGOPS international conference on Virtual execution environments. ACM, 2009, pp. 41-50.

[2] E. Feller, L. Rilling, and C. Morin, "Energy-aware ant colony based workload placement in clouds," in Proceedings of the 2011 IEEE/ACM 12th International Conference on Grid Computing. IEEE Computer Society, 2011, pp. 26-33.

[3] G. Von Laszewski, L. Wang, A. J. Younge, and X. He, "Power-aware scheduling of virtual machines in dvfs-enabled clusters," in Cluster Computing and Workshops, 2009. CLUSTER'09. IEEE International Conference on. IEEE, 2009, pp. 1-10.

[4] C.-M. Wu, R.-S. Chang, and H.-Y. Chan, "A green energy-efficient scheduling algorithm using the dvfs technique for cloud datacenters," Future Generation Computer Systems, vol. 37, 2014, pp. 141-147.

[5] E. Pinheiro, R. Bianchini, E. V. Carrera, and T. Heath, "Dynamic cluster reconfiguration for power and performance," in Compilers and operating systems for low power. Springer, 2003, pp. 75-93.

[6] J. S. Chase, D. C. Anderson, P. N. Thakar, A. M. Vahdat, and R. P. Doyle, "Managing energy and server resources in hosting centers," ACM SIGOPS operating systems review, vol. 35 , no. 5, 2001, pp. 103116.

[7] F. Clouet, S. Delamare, J.-P. Gelas, L. Lefèvre, L. Nussbaum, C. Parisot, L. Pouilloux, and F. Rossigneux, "A unified monitoring framework for energy consumption and network traffic," in TRIDENTCOMInternational Conference on Testbeds and Research Infrastructures for the Development of Networks \& Communities, 2015, p. 10.

[8] H. Sun, P. Stolf, and J.-M. Pierson, "Spatio-temporal thermal-aware scheduling for homogeneous high-performance computing datacenters," Future Generation Computer Systems, vol. 71, 2017, pp. 157-170.

[9] J. D. Moore, J. S. Chase, P. Ranganathan, and R. K. Sharma, "Making scheduling" cool": Temperature-aware workload placement in data centers." in USENIX annual technical conference, General Track, 2005, pp. 61-75.

[10] R. Bolze, F. Cappello, E. Caron, M. Daydé, F. Desprez, E. Jeannot, Y. Jégou, S. Lanteri, J. Leduc, N. Melab et al., "Grid'5000: A large scale and highly reconfigurable experimental grid testbed," The International Journal of High Performance Computing Applications, vol. 20, no. 4, 2006, pp. 481-494. 